\title{
A Reliability Optimization Algorithm for Wireless Sensor Network
}

\author{
https://doi.org/10.3991/ijoe.v14i06.8704 \\ Qiuming Zhang $\left({ }^{\square}\right)$ \\ China University of Geosciences, Hubei, China \\ qmzhang200811@yahoo.com \\ Jing Luo \\ Wuhan Vocational College of Software and Engineering, Hubei, China
}

\begin{abstract}
Aiming at the reliability optimization algorithm based on wireless sensor network, a data fusion algorithm based on extreme learning machine for wireless sensor network was proposed according to the temporal spatial correlation in data collection process. After analyzing the principles, design ideas and implementation steps of extreme learning machine algorithm, the performance and results were compared with traditional BP algorithm, LEACH algorithm and RBF algorithm in simulation environment. The simulation results showed that the data fusion optimization algorithm based on the limit learning machine for wireless sensor network was reliable. It improved the efficiency of fusion and the comprehensive reliability of the network. Thus, it can prolong the life cycle and reduce the total energy consumption of the network.
\end{abstract}

Keywords-wireless sensor network, optimization algorithm, extreme learning machine

\section{$1 \quad$ Introduction}

In sensor networks, a large number of sensor nodes are densely distributed and are randomly placed in the environment that needs to be measured. The measurement environment is usually an unreachable terrain or a disaster area. However, due to the damage of nodes, the exhaustion of energy and the change of environment, the sensor nodes are often unable to work normally, which leads to the change of the entire network topology. Wireless sensor networks collect data from monitoring area, integrate logical information world and objective physical world, and change the way of interaction between human and nature. Wireless sensor network has a lot of advantages, such as self-organization and the easy deployment. In addition, it does not require fixed network base support. It can be widely applied to scientific research, military reconnaissance, environmental monitoring, medical monitoring, agricultural breeding and other commercial areas, as well as special fields such as space exploration and disaster rescue. Wireless sensor networks are considered to be one of the most influ- 
ential technologies in the twenty-first Century and one of the top 10 technologies to change the world.

\section{State of the art}

In the face of complex industrial application environment, wireless sensor networks will be disturbed by the external environment in the process of data collection and data transmission. In addition, there are dynamic changes in the topology of the mobile network, which leads to the failure of the network. He, D. et al. [1] studied reliable wireless sensor network modeling and planning based on variable-length multi-objective optimization genetic algorithm. Sharawi, M. et al. [2] used the flower pollination optimization algorithm for global optimization of wireless sensor network life. Hoang, D. C. et al. [3] implemented an energy-efficient wireless sensor network clustering protocol based on a harmonious search algorithm in real time. Kim, J. Y. et al. [4] studied the cluster ant colony optimization algorithm for wireless sensor networks in dense environments. If the data collected by the sensor nodes cannot be passed to Sink effectively and quickly, it will affect the normal work, stability and reliability of the network. Attea, B. A. et al. [5] studied the reliability life of wireless sensor networks with multiple target disjoint sets. Karaboga, D. et al. [6] pointed out that according to the statistics at home and abroad, most of the existing WSN research focuses on routing protocol $(29 \%)$, MAC protocol $(19 \%)$, location algorithm $(17 \%)$ and energy efficiency $(30 \%)$. Only about $5 \%$ of the research is related to reliability. $\mathrm{Ma}, \mathrm{T}$. et al. [7] proposed a reliable information fusion algorithm based on wireless sensor networks. At present, there are few studies on the reliability and optimization algorithms of wireless sensor. This situation seriously restricts the development of the reliability technology of the Internet of things, mobile networks and wireless sensor networks. Iqbal, M. et al. [8] studied a multi-objective example of wireless sensor network optimization. Rajaram, M. L. et al. [9] analyzed the wireless sensor network simulation framework. Abdulhalim, M. F. and Attea, B. A. [10] studied the multilayer genetic algorithm coverage problem for the maximum separation of reliable sets in wireless sensor networks. To sum up, it is urgent to study the optimization algorithm of the reliability of wireless sensor networks. At present, the most important research work is to improve the reliability of mobile wireless sensor network node hardware devices, wireless communication links, data multi hop self-organization transmission and network operation, so as to ensure the long-term and stable work of the network. The research results will provide theory basis and technology guidance for the wireless sensor network reliability optimization design of nuclear radiation environment safety monitoring system, modern logistics warehousing environmental monitoring system, wearable medical monitoring system, large-scale agricultural mobile wireless sensor network and forest fire warning system.

To sum up, at present, the most important research work is to improve the reliability of mobile wireless sensor network node hardware devices, wireless communication links, data multi hop self-organization transmission and network operation. In order to solve this problem, a data fusion algorithm based on extreme learning ma- 
chine for wireless sensor network was proposed according to the temporal spatial correlation in data collection process. The method improved the efficiency of fusion and the comprehensive reliability of the network. Thus, it prolongs the life cycle and reduces the total energy consumption of the network.

\section{$3 \quad$ Method}

\subsection{Data fusion optimization algorithm for wireless sensor networks- extreme learning machine}

After analyzing the network energy consumption and network operation under different data fusion algorithms, a new data fusion method based on extreme learning machine for wireless sensor networks is proposed. The algorithm uses the extreme learning machine to learn, and the weights and thresholds of the neural network are obtained after optimization. The original data collected by the node are extracted, and the fusion processing is carried out. After that, the cluster head sends the fused data to the moving Sink. In this process, the data fusion in the network eliminates the transmission of redundant data and improves the accuracy of data fusion. The data fusion method proposed in this paper greatly reduces the feature dimension of the data, prolongs the network survival time, improves the efficiency of data fusion and the reliability of data transmission.

According to the different structure and function of the single hidden layer feedforward neural network, the extreme learning machine algorithms can be divided into three kinds: fixed extreme learning machine, incremental extreme learning machine and mixed extreme learning machine. According to their network structure, the three different classifications are mainly based on the number of nodes, the input layer and the number of the input layer nodes. Fixed extreme learning machine means that the number of hidden layer nodes of the neural network has been determined, and the output weights of all hidden layer neurons can be obtained by one calculation. The main feature of incremental extreme learning machine is that every time it is trained it will get an implicit layer of neuron weight. In the next training, neural network first obtains a new hidden layer neuron coefficient based on the last acquired weights and threshold network parameters. After training and learning, the new neuron weights are obtained by analysis and calculation. This iterative update can solve the "over saturation" phenomenon. The number of neurons in the hidden layer is acquired by experience and is difficult to determine. Mixed extreme learning machine is between the fixed and incremental extreme learning machines, and can also get better training speed. Compared with BP neural network, RBF neural network and support vector machine (SVM), it has the following advantages:

First, short learning time. In the course of learning and training, the input weight and threshold of the extreme learning machine are obtained in random way, and the hidden layer mapping function is known. In the whole process of learning and training, only the output weight of the network is calculated. Moreover, this value is only 
once calculated and does not need to modify the network weight. Therefore, the learning speed of the extreme learning machine is fast and the time is short.

Second, less parameter setting and algorithm implementation. This method is suitable for a variety of hidden layer mapping functions. The extreme learning machine algorithm only needs to set up one parameter of the network structure. The algorithm is simple and easy to be realized.

Third, good generalization ability. The extreme learning machine method does not cause the "over fitting" phenomenon to the training sample. In order to avoid the local optimal solution, its input weights and thresholds are obtained in a random way. These values have been calculated before the training data set is obtained and are independent of the training data.

Fourth, the hidden layer mapping function of extreme learning machine is much more than the traditional BP network gradient method. It can change the hidden layer mapping function, and does not need to change any algorithm. It only needs to change the hidden layer mapping function.

Although the extreme learning machine method has many advantages compared with the traditional machine learning algorithm, there are some disadvantages as well.

First, the input weights and the hidden layer thresholds of the ELM are generated randomly. If the inappropriate parameters are selected, it has a great impact on the generalization ability of the learning machine.

Second, when the training sample is not balanced or the number of training samples is small, the generalization performance of the algorithm is not ideal. Because of the characteristics of ELM's theory, generalization and training speed, many scholars have tried to use ELM algorithm to solve various problems of pattern recognition and classification in reality. In face recognition, text classification, medical diagnosis, image classification and other applications, the algorithm and model based on ELM have shown superior performance, which is superior to the traditional machine learning method and achieves satisfactory results.

\subsection{The basic principle of the extreme learning machine method}

The general network parameters of extreme learning machine algorithm include the input layer, the hidden layer and the number of nodes in the output layer, and the network weights and thresholds. It is assumed that the parameter $m, M$ and $n$ are the numbers of nodes in the network input layer, the hidden layer and the output layer. $g$

$(x)$ is an activation function of the neurons of the hidden layer. $b_{i}$ is the threshold. Assuming that there are $N$ different samples such as $\left(x_{i}, t_{i}\right)$ and $1 \leq i \leq N$. in the formula, the number of nodes in the input layer is $x_{i}=\left[\begin{array}{lll}x_{i 1}, & x_{i 2}, \ldots, x_{i m}\end{array}\right]^{\mathrm{T}} \in R^{m}$. The number of nodes in the output layer is $t_{i}=\left[\begin{array}{llll}t_{i 1}, & t_{i 2}, & \ldots, & t_{i n}\end{array}\right]^{\mathrm{T}} \in R^{n}$.

The network model of the extreme learning machine can be expressed in mathematical expressions as:

$$
\sum_{i=1}^{L} \beta_{i} g\left(\omega_{i} \bullet x_{i}+b_{i}\right)=O_{j}, j=1,2, \cdots, N
$$


In the formula, $\omega_{i}=\left[\omega_{i 1}, \omega_{i 2}, \cdots, \omega_{i m}\right]^{T}$ represents the input weight vector between the connection network input layer and the $i$-th hidden layer node. $\beta_{i}=\left[\beta_{i 1}, \beta_{i 2}, \cdots, \beta_{i n}\right]^{T}$ represents the output weight vector connecting the $i$-th hidden layer node and the network input layer node. $O_{i}=\left[O_{i 1}, O_{i 2}, \cdots, O_{i n}\right]^{T}$ represents the network output value.

The cost function $E$ of the extreme learning machine is expressed as:

$$
E(S, \beta)=\sum_{j=1}^{N}\left\|O_{j}-t_{j}\right\|
$$

In the formula, $S=\left(\omega_{i}, b_{i}, i=1,2, \cdots, M\right), \omega_{i}$ and $b_{i}$ are network input weights and hidden layer node thresholds, respectively. According to the extreme learning machine method pointed by Professor Huang in Singapore Nanyang Technology University, the training goal of extreme learning machine network is to find optimal $\mathrm{S}$ and $\beta$ to ensure that the error between the output value of the neural network and the corresponding actual value is minimal. The mathematical expression is $\min (\mathrm{E}(\mathrm{S}, \beta))$.

\subsection{Design of reliability algorithm for data transmission in Wireless Sensor Networks}

Because of the limited performance of the sensor nodes and the disturbance of the external environment, a single sensor node can only obtain local limited and uncertain information. In order to eliminate the influence of the external environment and the uncertainty of collected information, data fusion technology combines, analyzes, processes and integrates monitoring information from different sources, information modes and expression ways from multiple sensing nodes, and extracts more precise comprehensive information. Thus, it is convenient for users to understand the overall situation correctly and provide the basis for the correct decision. Data fusion technology has become an important means for wireless sensor networks to save energy, improve work efficiency and data transmission reliability and prolong network lifetime. In order to simplify the network simulation calculation, the information of the node and topology of the wireless sensor network is assumed.

The technology of data fusion is to process the large amount of raw data collected by the wireless sensor network nodes. Redundant information can be suppressed to reduce the enormous pressure on the wireless sensor networks with limited energy, storage capacity and limited network bandwidth. In order to meet the requirements of performance, only a small amount of information is needed to save network energy. The flow chart of the mobile wireless sensor network data fusion is shown in figure 1 . A single sensor node first collects data. After the data preprocessing, it is transmitted to the cluster head node by route multi-hop and self-organization. The cluster head 
node then converts the data of the received multiple sensor data in the network, and then sends the output data to the Sink node.

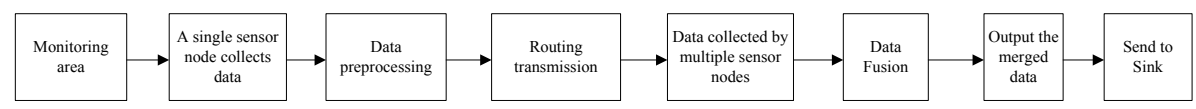

Fig. 1. Mobile wireless sensor network data fusion process

\subsection{Algorithm design idea}

Combining the spatiotemporal correlation of data collection, the data fusion method is applied to the fusion of cluster heads and intra cluster nodes in the wireless sensor network. A new method of artificial intelligence extreme learning machine is introduced on the basis of routing clustering algorithm, and a data fusion method based on extreme learning machine is proposed. The collected data are analyzed and processed synthetically, and the data with poor reliability, invalid and redundant are deleted to improve the accuracy and accuracy of the data. It solves the problem of data fusion efficiency, network life cycle and data transmission reliability. At the same time, the amount of data transmission and the energy consumption of the network are reduced and the work efficiency of the network is improved.

The extreme learning method data fusion (ELMDF) algorithm is developed on the basis of classic clustering LEACH algorithm for mobile wireless sensor networks. The classic clustering LEACH routing protocol first clusters all nodes in the detection area and then assigns random values between 0 and 1 . If the value is less than a threshold, then the node becomes a cluster head. The cluster head and the nodes of the family members form a stable cluster structure. The cluster member nodes preprocess the collected data and then transmit them to the cluster heads. The cluster head combines the collected data and then removes redundant and useless information, and then forward it to the Sink. The ELMDF method is to use the extreme learning machine method to deal with the data between the cluster members and the cluster heads.

\subsection{Algorithm implementation}

First, all the components of the wireless sensor network are initialized to determine the state of the common nodes, routing nodes and cluster head nodes in the network. Then, according to the location of the node, the cluster structure of the monitoring area is set up. The cluster head nodes are selected randomly in the cluster, and all the information of the cluster members can be obtained through the cluster head nodes. When the clustering of the wireless sensor networks is stable, the routing table from the common node to the Sink is constructed. The cluster head node forwards the information of the cluster member node to the Sink node. After the network work is stable, we first need to train the wireless sensor data fusion model, so as to get the number of nodes, weights and threshold parameters of the hidden layer in the extreme learning machine network. Due to the limited energy of the common sensor nodes in wireless sensor networks, data perception and sending / receiving tasks have to be 
performed. In order to reduce the energy consumption of common nodes and prolong the lifetime of the network, the training of the data fusion model based on the extreme learning machine is completed within the Sink node of the mobile sensor network. The Sink node constructs the extreme learning machine network structure according to the received information, and collects samples that are matched with the members' information in the cluster head database to train the network. After the training is completed, the relevant information of the related data fusion model is obtained. After that, the Sink node sends network parameters (the number of hidden layer nodes, network weights and thresholds) in the data fusion model to the corresponding cluster head nodes. The cluster head is used to fuse and extract the data from the members of the cluster according to the trained data fusion model. At the same time, redundant and useless information are deleted, and the data after the fusion is temporarily stored. When the mobile Sink reaches the cluster head communication range, it is forwarded to the Sink.

Data fusion steps of wireless sensor networks based on extreme learning machines: First, the optimal data fusion model based on the extreme learning machine method is constructed. Then, the fusion model is applied to the cluster structure of wireless sensor network, which improves the accuracy of the network fusion, reduces the data transmission and prolongs the network life. The flow chart of the ELMDF based wireless sensor network data fusion algorithm is shown in figure 2.

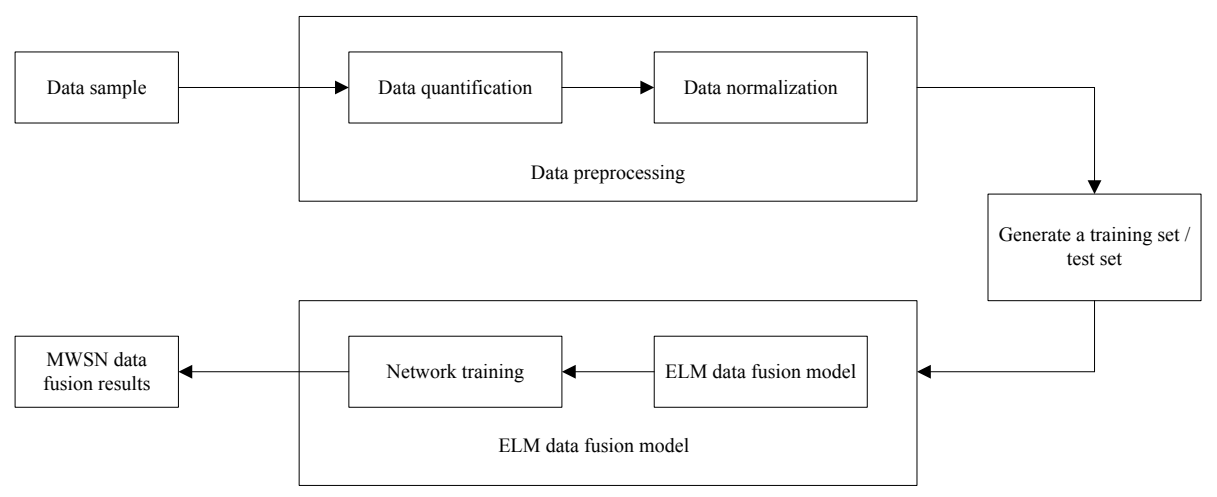

Fig. 2. Wireless sensor network data fusion process based on extreme learning machine

The implementation steps of the wireless sensor network data fusion based on the extreme learning machine are as follows:

Step 1: The parameters of the model are initialized, such as the security verification of the node, the initialization of the network, the location of the node and the number of the ELM hidden layer nodes;

Step 2: The data fusion model is initialized under the constraints of the input weight and threshold;

Step 3: The data fusion model and the ELM network model are mapped one by one, and the predicted error is used as the optimization function of the data fusion; 
Step 4: The cluster head is selected from the common node and the cluster head perception area is established;

Step 5: The cluster head sends the cluster head member node information table to the Sink node;

Step 6: The Sink node constructs the ELM data fusion model and trains the sample data to obtain the network parameters of the extreme learning machine (weights and thresholds);

Step 7: If the terminating condition of the network training is not reached, it will continue to execute;

Step 8: The Sink node sends the trained ELM network structure parameters to the corresponding nodes.

Step 9: The cluster head node combines the received information with the trained ELM network structure, and then sends the fusion data to the Sink node;

Step 10: The network reliability data transmission based on data fusion ends at this time.

\section{$4 \quad$ Results}

In order to prove the optimization performance of the algorithm, the ELM algorithm is mainly compared with the traditional BP, LEACH and RBF algorithms. By comparing the data fusion efficiency, the total network energy consumption and the network life cycle, it is proved that the data fusion algorithm based on extreme learning machine in mobile wireless sensor network is a more optimized algorithm.

\subsection{Simulation environment setting}

In order to test the performance of the data fusion algorithm in this paper, the experiment and performance analysis are carried out by MATLAB2014b simulation software. Assuming that the wireless sensor network nodes were randomly distributed in the two-dimensional space of $1000 \times 1000 \mathrm{~m}^{2}$. The number of sensor nodes is 300 . The number of simulation polling is set to 500. In each simulation, the sensor nodes have 10 packets from the source node to the mobile Sink. The capacity of each packet is $4 \mathrm{~KB}$. The initial energy of mobile Sink is $50 \mathrm{~J}$, and Sink moves in a straight line at a speed of $5 \mathrm{~m} / \mathrm{s}$. The parameter of extreme learning machine is set as follows: the number of initial hidden layer nodes is 10 , and the number of hidden layer nodes increases with a period of 20 until the maximum set value is 300 . The hidden layer activation function of ELM selects the hardlim function for learning. In order to enhance the persuasiveness of the model, the results presented in this paper are the average results of the 100 experiments. The simulation environment parameters are set as shown in table 1 . 
Table 1. Simulation environment parameter settings

\begin{tabular}{|c|c|}
\hline Variable & Value \\
\hline Network range & $1000 \times 1000 \mathrm{~m}^{2}$ \\
\hline Number of nodes & 300 \\
\hline Communication radius & $80 \mathrm{~m}$ \\
\hline$V_{\text {sink }}$ & $5 \mathrm{~m} / \mathrm{s}$ \\
\hline Initial energy & $1 \mathrm{~J}$ \\
\hline$E_{\text {elec }}$ & $50 \mathrm{nj} / \mathrm{bit}$ \\
\hline$E_{f s}$ & $10 \mathrm{pJ} / \mathrm{bit} / \mathrm{m}^{2}$ \\
\hline$E_{m p}$ & $0.0013 \mathrm{pJ} / \mathrm{bit} / \mathrm{m}^{4}$ \\
\hline$l$ & $4000 \mathrm{bits}$ \\
\hline$d_{0}$ & $\sqrt{E_{f S} / E_{m p}}=87 \mathrm{~m}$ \\
\hline
\end{tabular}

In order to better illustrate the superiority of the algorithm, the LEACH algorithm, BP neural network, RBF neural network and extreme learning machine algorithm are compared.

LEACH algorithm: data fusion algorithm for mobile wireless sensor networks based on clustering;

BP neural network: data fusion algorithm for mobile wireless sensor network based on BP neural network;

RBF neural network: data fusion algorithm for mobile wireless sensor network based on RBF neural network;

Extreme learning machine algorithm: data fusion algorithm for mobile wireless sensor network based on extreme learning machine

\subsection{Comparison of data fusion efficiency}

The efficiency of data fusion shows the fusion performance of the data fusion algorithm. When the efficiency is high, the network data transmission, the energy consumption and the packet loss rate are less. At the same time, the network life and reliability are high. Therefore, the efficiency of data fusion is one of the important performance evaluations of this paper. The data fusion rate is calculated as follows: assuming that sensor node $i$ forward the perceived data to the sensor node $j$. According to the time and spatial correlation of data acquisition, the formula of data correlation coefficient is:

$$
\rho(i, j)=1-H(i \mid j) / H(i)
$$

Parameter $\mathrm{H}(i)$ represents the size of the data sent by the perceptive node $i$. Parameter $\mathrm{H}(\mathrm{i} \mid \mathrm{j})$ indicates that the receiving node $j$ removes the redundant information associated with the original data based on the local data and the spatiotemporal correlation of data, and gets the size of the fusion data. The data correlation system 
$\rho(i, j)$ reflects the degree of correlation between the data, and the data related system $\rho(i, j)$ is inversely proportional to the distance between the sensor nodes. The calculation formula is as follows:

$$
\rho(i, j)=1 /[1+d(i, j)\rfloor
$$

Data fusion rate is made up of two parts. Part of the data fusion is transmitted from sensor nodes and other nodes around them. The other part is the fusion of the original data collected by the upstream node and the data collected by the cluster head itself. Figure 3 is a comparison diagram of the data fusion efficiency of the four algorithms.

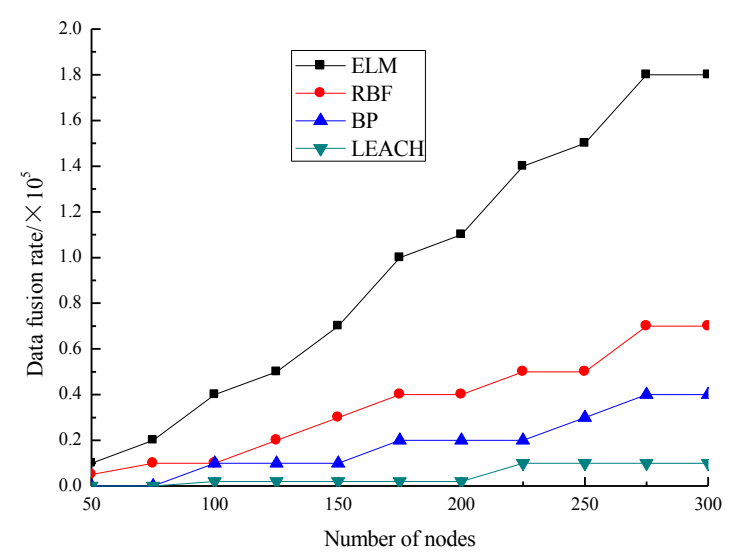

Fig. 3. Comparison of data fusion efficiency

As shown in figure 3, with the increase of the number of network nodes, the data fusion rate of LEACH routing protocol, BP neural network and RBF neural network data fusion algorithm is increasing. The algorithm proposed in this paper has the largest increase range, showing a straight-line upward trend. The reason is that with the increase of the number of sensor nodes, a lot of neighbor nodes will be added between nodes. The number of neighbor nodes of the data collected by the perceptive node increases, and the probability of data fusion also increases. Compared with the data fusion rate of ELM and LEACH, BP neural network and RBF neural network, the calculation results are $94.4 \%, 79.1 \%$ and $64.6 \%$ respectively.

\subsection{Comparison of total energy consumption in network}

The total energy consumption of the sensor network is defined as the sum of energy consumed by the whole network sensor node. When the total energy consumption of the network becomes small, the fusion efficiency of the network data is higher, the data of the transmission is less and the life cycle of the network becomes longer. The total energy consumption of the sensor is used as a measure index, and the total energy consumption of the four algorithms is shown in figure 4 . 


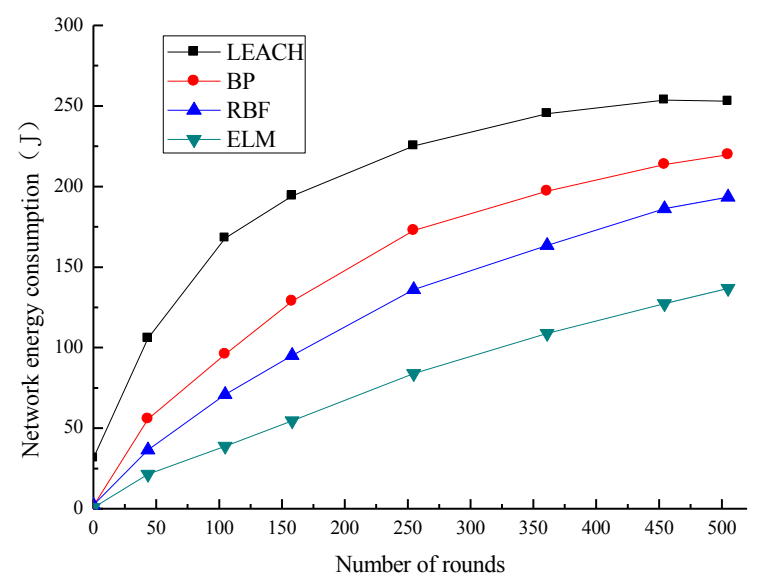

Fig. 4. Comparison of total network energy consumption

As shown in figure 4, with the increase of the round number, the network energy consumption of the four algorithms is increasing gradually. However, the growth of the LEACH algorithm is the largest, and the algorithm proposed in this paper has the smallest increase. In addition, the energy consumption of the LEACH routing protocol is the largest and the BP neural network is larger. The RBF neural network is smaller, and the extreme learning machine algorithm is the smallest. With 400 round numbers as reference, the algorithm is compared with BP neural network and RBF neural network in the LEACH protocol and the accuracy of the algorithm is increased by $32.8 \%, 22.8 \%$ and $12.8 \%$ respectively. In the process of data transmission, the LEACH algorithm has no data fusion. As time goes on, energy consumption increases sharply. BP neural network, RBF neural network and extreme learning machine algorithm use data fusion to reduce the amount of data transmission and the energy consumption of nodes. From the comparison of the network energy consumption of the three algorithms, it shows that the proposed algorithm has the advantages of low network parameters setting and high learning rate, which reduces the energy consumption of the network.

\subsection{Network life cycle comparison}

The life cycle of the network is one of the most important indicators of the performance of wireless sensor networks. When the life cycle of the network is long, it is proved that the network can perform monitoring tasks longer and avoid frequent replacement of batteries. By comparing with the alternatives in other literature, Figure 5 performs a performance evaluation of the proposed data fusion algorithm. The graph and change on the number of living nodes of different data fusion algorithms with increasing cycles are compared. 


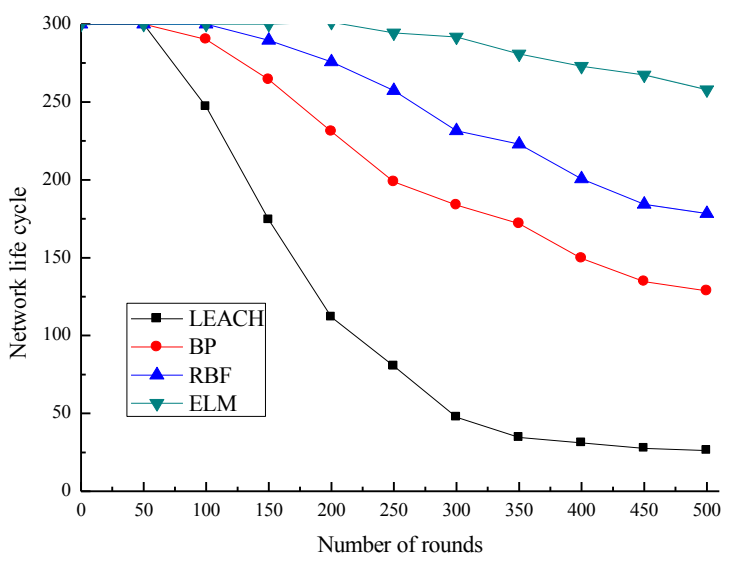

Fig. 5. Network life cycle

As shown in figure 5, in the LEACH protocol, the death time of 50\% sensor nodes is in 178th round. In the data fusion of BP neural network, the death time of $50 \%$ sensor nodes is $402^{\text {nd }}$ round. When the RBF neural network algorithm runs 500 rounds, the death rate of the node is $43.3 \%$, and the algorithm proposed in this paper is $16.7 \%$. The algorithm proposed in this paper takes into account the residual energy and the distance to the sink node in the wireless sensor network, and transfers the sensing data of each node to the sink node through the family header node. The energy consumption of the network is small. In the case of 500 rounds, the network life cycle is compared to the LEACH protocol. The BP neural network algorithm and the $\mathrm{RBF}$ neural network algorithm are improved by $87.3 \%, 38.3 \%$ and $26.2 \%$ respectively.

\section{Conclusions}

How to efficiently and quickly integrate data, improve the efficiency of fusion and resource utilization, and reduce network energy consumption, data fusion is one of the key technologies for wireless sensor networks. In the process of redundant data transmission, too much energy will be consumed. It is necessary to shorten the lifetime of the network and improve the reliability of data transmission. Through this study, the following conclusions are drawn.

(1) According to the temporal and spatial correlation in data collection process of wireless sensor network, a data fusion algorithm based on extreme learning machine in wireless sensor network is proposed.

(2) The generalization performance of the extreme learning machine is used. The adjustable parameters are relatively small, and the learning ability is strong. The characteristics of the original data are extracted and the data fusion is carried out.

(3) The results show that the algorithm proposed in this paper can improve the efficiency of fusion and the comprehensive reliability of the network. In addition, it can prolong the life cycle and reduce the total energy consumption of the network. 


\section{References}

[1] He, D., Mujica, G., Portilla, J., \& Riesgo, T. Modelling and planning reliable wireless sensor networks based on multi-objective optimization genetic algorithm with changeable length. Journal of Heuristics, 2015, vol. 21(2), pp. 257-300. https://doi.org/10.1007/ s10732-014-9261-2

[2] Sharawi, M., Emary, E., Saroit, I. A., \& El-Mahdy, H. Flower pollination optimization algorithm for wireless sensor network lifetime global optimization. International Journal of Soft Computing and Engineering, 2014, vol. 4(3), pp. 54-59.

[3] Hoang, D. C., Yadav, P., Kumar, R., \& Panda, S. K. Real-time implementation of a harmony search algorithm-based clustering protocol for energy-efficient wireless sensor networks. IEEE transactions on industrial informatics, 2014, vol. 10(1), pp. 774-783. https://doi.org/10.1109/TII.2013.2273739

[4] Kim, J. Y., Sharma, T., Kumar, B., Tomar, G. S., Berry, K., \& Lee, W. H. Intercluster ant colony optimization algorithm for wireless sensor network in dense environment. International Journal of distributed sensor networks, 2014, vol. 10(4), pp. 457402. https://doi.org/10.1155/2014/457402

[5] Attea, B. A., Khalil, E. A., Özdemir, S., \& Y1ldız, O. A multi-objective disjoint set covers for reliable lifetime maximization of wireless sensor networks. Wireless Personal Communications an International Journal, 2015, vol. 81(2), pp. 819-838. https://doi.org/10.1007/s11277-014-2159-3

[6] Karaboga, D., Gorkemli, B., Ozturk, C., \& Karaboga, N. A comprehensive survey: artificial bee colony (ABC) algorithm and applications. Artificial Intelligence Review, 2014, vol. 42(1), 21-57. https://doi.org/10.1007/s10462-012-9328-0

[7] Ma, T., Liu, Y., Fu, J., \& Jing, Y. A reliable information fusion algorithm for reputation based wireless sensor networks. International Journal of Future Generation Communication \& Networking, 2015, vol. 8(1), pp. 114-118.

[8] Iqbal, M., Naeem, M., Anpalagan, A., Ahmed, A., \& Azam, M. Wireless sensor network optimization: multi-objective paradigm. Sensors, 2015, vol. 15(7), pp. 17572-17620. https://doi.org/10.3390/s150717572

[9] Rajaram, M. L., Kougianos, E., Mohanty, S. P., \& Choppali, U. Wireless sensor network simulation frameworks: A tutorial review: MATLAB/Simulink bests the rest. IEEE Consumer Electronics Magazine, 2016, vol. 5(2), pp. 63-69. https://doi.org/10.1109/ MCE.2016.2519051

[10] Abdulhalim, M. F., \& Attea, B. A. Multi-layer genetic algorithm for maximum disjoint reliable set covers problem in wireless sensor networks. Wireless Personal Communications, 2015, vol. 80(1), pp. 203-227. https://doi.org/10.1007/s11277-014-2004-8

\section{$7 \quad$ Authors}

Qiuming Zhang is with China University of Geosciences, Hubei, China.

Jing Luo is with the Practice training centre, Wuhan Vocational College of Software and Engineering, Hubei, China.

Article submitted 27 February 2018. Resubmitted 26 March 2018. Final acceptance 16 April 2018. Final version oublished as submitted by the authors. 